\author{
Michael Scharl ${ }^{a}$ \\ Gerhard Rogler ${ }^{a}$ \\ Luc Biedermann ${ }^{a}$
}

\title{
Anthocyane, Heidelbeeren und Curcuma: Wirksame Therapeutika bei Darmentzündungen?
}

\section{Einleitung}

Die Diskussion über eine gesunde und ausgewogene Ernährung in der Bevölkerung gewinnt zunehmend an Bedeutung. Berichte über positive wie auch negative Auswirkungen bestimmter Diäten und Ernährungsformen, aber auch über Nahrungsmittel und Nahrungsmittelzusatzstoffe gewinnen eine zunehmende öffentliche Aufmerksamkeit. Ein Beispiel hierfür sind gastrointestinale Beschwerden, wie etwa die funktionellen Magen-DarmErkrankungen, für die im Internet und auch in Druckmedien ein breites Spektrum an diätetischen Interventionen empfohlen wird. In den letzten Jahren hat hier insbesondere die FODMAPDiät eine prominente Stellung erreicht. Allgemein bekannt sind ebenfalls Nahrungsmittelunverträglichkeiten, wie die Laktoseintoleranz oder FructoseMalabsorption, die sich insbesondere durch gastrointestinale Beschwerden, wie Diarrhö oder Blähungen, äussern.

Nahrungsmittelunverträglichkeiten dürfen jedoch nicht mit Nahrungsmittelallergien verwechselt werden. Echte Nahrungsmittelallergien sind $\operatorname{IgE}(\mathrm{Immunglobulin} \mathrm{E})$-vermittelte Reaktionen des Immunsystems auf Nahrungsbestandteile, wie z.B. Teile von Erdnüssen oder bestimmten Schalenfrüchten. Typischerweise macht sich eine Nahrungsmittelallergie mit einer Schleimhautschwellung nach der Aufnahme der allergenen Subs-

${ }^{a}$ Klinik für Gastroenterologie und Hepatologie, Universitätsspital Zürich, Zürich, Schweiz $\tan z$ bemerkbar. Diese Schwellungen können im Mund-, Nasen- und Rachenraum, aber auch im MagenDarm-Trakt oder in den Atemwegen auftreten und, je nach Ausprägung, teils sogar lebensbedrohlich werden. Des Weiteren ist die Glutenunverträglichkeit, die Zöliakie oder Sprue, bekannt. Hierbei kommt es zu einer Immunreaktion des Körpers gegen Bestandteile des Weizeneiweisses Gluten. Als Folge dieser Reaktion, die Merkmale einer Allergie wie auch einer Autoimmunerkrankung aufweist, entsteht eine chronische Dünndarmentzündung.

Neben diesen zahlreichen Beispielen von negativen Auswirkungen bestimmter Ernährungsbestandteile gibt es jedoch auch zunehmend Berichte über positive und therapeutische Wirkungen von Nahrungsbestandteilen. Auch hierfür kann als Beispielorgan der Magen-Darm-Trakt genannt werden. Insbesondere bei chronischen Darmerkrankungen gibt es zunehmende Evidenz, dass Nahrungsbestandteile bzw. Nahrungsmittelzusätze eine antientzündliche Wirkung aufweisen [1].

\section{Heidelbeeren und Anthocyane als Heilmittel}

Ein prominentes Beispiel einer solchen antientzündlich wirksamen, pflanzlichen Substanz sind die Polyphenole. Chemisch gesehen bestehen sie aus mindestens einem aromatischen Polyphenolring, an den wenigstens eine Hydroxylgruppe angeheftet ist. Aufgrund ihrer chemischen Beschaffenheit können die Polyphenole in zwei Gruppen, d.h. die Flavonoide und die Nicht-Flavonoide, unterschieden werden. Eine wichtige Untergruppe der in der Nahrung enthaltenen Flavonoide sind die Anthocyanidine. Diese sind in vielen Früchten, in Gemüse wie etwa Auberginen und in Blüten enthalten und bewirken dort deren typische rote, blaue oder violette Färbung. In Pflanzen sind die Anthocyanidine meist an Zucker oder organische Säuren gekoppelt und werden dann als Anthocyane bezeichnet [2,3].

Unsere gewöhnliche Nahrung ist bereits reich an natürlichen Phenolen. Insbesondere Beeren, Rotwein, Blattund Wurzelgemüse sowie bestimmte Vollkornprodukte enthalten eine hohe Konzentration an Anthocyanen. Allerdings ist die tägliche Aufnahme der Anthocyane mit der Nahrung sehr variabel. Eine Diät, welche insbesondere aus Auberginen, dunklen Trauben und Beeren besteht, dürfte den höchsten Gehalt an Anthocyanen aufweisen - mit einer täglichen Menge von über $100 \mathrm{mg}$ [4]. Aufgrund ihrer mehrfach nachgewiesenen gesundheitsfördernden Wirkung nimmt das öffentliche Interesse an den Anthocyanen stetig zu [5]. Verschiedene Berichte zeigen, dass Anthocyane eine antientzündliche, antioxidative, antikarzinogene, antimikrobielle sowie auch neuroprotektive Wirkung haben $[2,3,6]$. In verschiedenen epidemiologischen Studien wurde nachgewiesen, dass eine an Polyphenolen reiche Ernährung vor dem Auftreten von koronarer Herzerkrankung, verschiede-

\section{KARGER}

(C) 2017 S. Karger GmbH, Freiburg

Fax +497614520714
PD Dr. Michael Scharl

Klinik für Gastroenterologie und Hepatologie

Universitätsspital Zürich

Rämistrasse 100, 8091 Zürich, Schweiz

michael.scharl@usz.ch 
nen Tumorerkrankungen, aber auch chronisch-entzündlichen Darmerkrankungen schützen könnte [7-9].

Als Ursache dieser gesundheitsfördernden Auswirkungen der Anthocyane wird zum einen die aufgrund ihrer Phenolstruktur bestehende antioxidative Wirkung angenommen, da sie in der Lage sind, reaktive Sauerstoffspezies zu inaktivieren [10]. Die Risikoreduktion für das Auftreten der koronaren Herzerkrankung durch Anthocyane scheint insbesondere durch die Hemmung der LDL(Low-Density Lipoprotein)-Oxidation zu erfolgen [11]. Zudem wirken Anthocyane antimikrobiell, z.B. gegen Helicobacter pylori und Bacillus cereus, und verringern im Mausmodell die Expression verschiedener Gene, die an der Entstehung der Atherosklerose beteiligt sind $[6,12]$. Zudem hemmen Anthocyane entzündungsfördernde Signalwege in den Abwehrzellen. Sie sind Hemmstoffe der 5-Lipoxygenase, welche für die Entstehung entzündungsfördernder Moleküle wichtig ist, und verringern die Produktion der Cyclooxygenase 2 (COX-2). Zudem reduzieren sie die Sekretion von entzündungsfördernden Botenstoffen sowie die Aktivität von entsprechenden Signalmolekülen in Immunzellen [13-17]. Unsere Arbeitsgruppe konnte zeigen, dass ein Anthocyan-enthaltender Heidelbeerextrakt in der Lage ist, die entzündungsfördernde Wirkung der Zytokine Tumornekrosefaktor (TNF) und Interferon gamma (IFN $\gamma$ ) in menschlichen Immunzellen abzuschwächen. Der Heidelbeerextrakt verringerte hierbei die Aktivierung von Signalmolekülen ebenso effektiv wie die Expression und Sekretion von weiteren entzündungsfördernden Zytokinen [18].

In einer randomisierten Studie mit Patienten, die ein erhöhtes Risiko für kardiovaskuläre Ereignisse aufwiesen, verringerte sich der Plasmaspiegel mehrerer Entzündungsmarker, wie etwa C-reaktives Protein (CRP) und Interleukin (IL) 6, in der Gruppe der Patienten, die einen Heidelbeersaft erhielten, signifikant [19]. In einer wei- teren Untersuchung bewirkte der Konsum eines Heidelbeersaftes einen Rückgang des postprandialen Insulinbedarfs. Eine weitere Pilotstudie ergab Hinweise, dass der Verzehr von Heidelbeeren eventuell sogar vor dem Auftreten von Dickdarmkrebs schützen könnte [20,21].

\section{Anthocyane und chronische Darmentzündungen}

In verschiedenen In-vivo-Studien mit Mäusen wurde nachgewiesen, dass Anthocyane aus Heidelbeeren und schwarzen Himbeeren die Symptome einer Darmentzündung deutlich verringern können [22-24]. Eine Studie aus unserer Arbeitsgruppe konnte insbesondere zeigen, dass getrocknete Heidelbeeren als Quelle für Anthocyane ebenso wie ein 1\%iger sowie ein 10\%iger Anthocyan-Extrakt, der Mäusen mit der regulären Nahrung verabreicht wurde, die Symptome und Auswirkungen einer akuten Darmentzündung deutlich verringern. Auch bei einer chronischen Form einer Darmentzündung waren getrocknete Heidelbeeren in der Lage, die Symptome und klinischen Folgen im Mausmodell zu lindern. Diese Beobachtung konnte sowohl mittels histologischer Analyse des Mausdarmes als auch aufgrund reduzierter Spiegel entzündungsfördernder Zytokine untermauert werden [24]. Aufgrund dieser positiven Daten aus den Tierexperimenten sowie aufgrund der Erkenntnisse aus Studien mit verschiedenen Patientengruppen entschlossen wir uns, eine klinische Pilotstudie zur Wirksamkeit und Sicherheit von Anthocyan-reichem Heidelbeerextrakt bei Patienten mit einer speziellen Form der chronisch-entzündlichen Darmerkrankungen, der sogenannten Colitis ulcerosa, durchzuführen [25].

Die Colitis ulcerosa ist eine chronische Darmentzündung, die zeitlebens in rezidivierenden Krankheitsschüben verlaufen kann und hauptsächlich junge Erwachsene betrifft. Es handelt sich um eine Entzündung des Dickdarmes, welche bei jedem Patienten unterschiedlich stark ausgeprägt ist. Die Hauptsymptome sind schwere, oftmals blutige Durchfälle und Bauchschmerzen. Viele Patienten leiden an rezidivierenden Schüben, die unterschiedlich stark ausgeprägt sein können, jedoch häufig die Lebensqualität der betroffenen Patienten deutlich einschränken. Rund zwei Drittel der Patienten können mittels einer Therapie mit Mesalazin (5-Aminosalicylsäure) suffizient behandelt werden. Die Behandlung des verbleibenden Drittels der Patienten stellt jedoch häufig ein schwieriges medizinisches Problem dar, und rund $10 \%$ aller Colitis-ulcerosa-Patienten müssen sich letztlich sogar einer Kolektomie unterziehen. Es gibt zwar auch für die schwereren Fälle verschiedene Medikamente, die teils gute Wirkungen erzielen. Hierzu gehören insbesondere Kortikosteroide, anti-TNF-Antikörper, anti-Integrin-Antikörper und Azathioprin. Allerdings haben diese Medikamente oftmals signifikante Nebenwirkungen, sodass sie meist keine optimale Alternative für eine Langzeittherapie darstellen. Zudem sind insbesondere die Antikörper, also die Biologika, sehr teuer - und auch diese Medikamente wirken häufig nur bei einem Teil der Patienten.

Somit ist es offensichtlich, dass neue Medikamente, die zum einen zwar wirksam, zum anderen aber auch gut verträglich und kostengünstig sind, dringend benötigt werden. Ein Wirkstoff, der dieses Profil optimal erfüllen könnte, wären die Anthocyane. Um die Wirksamkeit und Sicherheit der Anthocyane bei Patienten mit Colitis ulcerosa zu untersuchen, haben wir eine klinische Pilotstudie mit einer Überwachungsphase von insgesamt 9 Wochen durchgeführt [25]. In diesem Rahmen testeten wir eine täglich verabreichte, standardisierte, Anthocyan-reiche Heidelbeerzubereitung bei 13 Patienten mit leichter bis mittelschwerer Colitis ulcerosa. Am Ende der sechswöchigen Behandlungsperi- 
ode erreichten rund zwei Drittel der Patienten eine klinische Remission, die mittels des Colitis-Aktivitätsindex (CAI) standardisiert gemessen wurde, und erfüllten somit den primären Studienendpunkt. Über 90\% der Patienten erreichten eine klinische Verbesserung ihrer Symptome. Die in den Fäzes gemessenen Werte des Calprotectin, eines Proteins, das als Marker für das Ausmass der Entzündung im Darm gilt, sanken bei allen Patienten unter der Einnahme der Heidelbeerzubereitung. Unter der Therapie mit Anthocyanen ergab sich eine deutliche Reduktion der makroskopisch wie mikroskopisch nachweisbaren Entzündung im Darm. Dies zeigte sich mithilfe einer Darmspiegelung und der anschliessenden histopathologischen Analyse von dabei entnommenen Darmbiopsien [25]. Bei weiteren Untersuchungen dieser Gewebeproben zeigte sich eine Verminderung der entzündungsfördernden Zytokine TNF und IFN $\gamma$ sowie des Signalmoleküls nukleärer Faktor kappa B (NF$\kappa B)$. Interessanterweise konnte in den Biopsien derjenigen Patienten, die gut auf die Therapie ansprachen, eine $\mathrm{Zu}$ nahme der hauptsächlich antientzündlich wirksamen Zytokine IL-10 und IL-22 nachgewiesen werden. Zudem fand sich im Serum dieser Patienten eine Abnahme der entzündungsfördernden Zytokine TNF und MCP-1. Diese Beobachtungen konnten bei Patienten, welche nicht suffizient auf die Behandlung ansprachen, nicht gemacht werden [26]. Bei vielen Patienten fand sich 2 Wochen nach Beendigung der Anthocyan-Einnahme jedoch ein Wiederanstieg des Entzündungsmarkers Calprotectin. Während der gesamten Studie traten keine Nebenwirkungen auf. Diese Studie wies somit erstmals die Wirksamkeit einer standardisierten, Anthocyanreichen Heidelbeerzubereitung bei Patienten mit Colitis ulcerosa nach [25].

Aufgrund dieser Studie ermöglicht uns der Schweizer Nationalfonds nun eine placebokontrollierte, randomi- sierte, multizentrische Studie bei Patienten mit Colitis ulcerosa, die demnächst in der Schweiz und in Deutschland beginnen wird. Wir hoffen, dass wir hier genügend Patienten finden werden, die anstelle der üblichen Therapien bereit sind, an dieser Studie mitzuwirken.

\section{Curcuma bei chronischen Darmentzündungen}

Eine weitere Pflanze, der antientzündliche Wirkungen insbesondere im Magen-Darm-Trakt bescheinigt wurden, ist die Gelbwurz, Turmeric oder Curcuma. Gemahlene Curcuma ist seit vielen Tausend Jahren ein wichtiger Bestandteil der verschiedenen Curry-Präparationen in Indien. Curcumin ist auch seit Jahrhunderten in der ayurvedischen und Traditionellen Chinesischen Medizin weit verbreitet, um eine breite Palette von entzündlichen Erkrankungen zu behandeln. Invitro-Experimente haben die entzündungshemmenden und antioxidativen Eigenschaften von Curcumin in menschlichen Lymphozyten und Darmepithelzelllinien gezeigt [27-34]. Curcumin-Präparationen waren auch in der Lage, in vivo die murine experimentelle Colitis abzumildern [33, 34]. Vor Kurzem zeigten Hanai et al. [35], dass Curcumin bei der Erhaltung der Remission für bis zu 12 Monate bei Patienten mit Colitis ulcerosa gegenüber Placebo deutlich überlegen war.

Auch zur Behandlung des akuten Colitis-Schubes ist Curcumin erstaunlich effektiv, wie die Arbeitsgruppe um Ben-Horin in Tel Aviv kürzlich zeigte: Die Arbeitsgruppe führte eine multizentrische, randomisierte, placebokontrollierte, doppelblinde Studie an 50 mit 5-ASA behandelten Patienten mit aktiver Colitis ulcerosa durch. Die Patienten wurden nach dem Zufallsprinzip den beiden Gruppen zugeteilt, die für 1 Monat (unter Weiterführung der 5-ASA-Therapie) entweder Placebo oder Curcumin-Kapseln (3 g/Tag) erhielten. 14 Patienten
(53,8\%), die Curcumin erhielten, erreichten nach 4 Wochen eine klinische Remission, jedoch keiner der PlaceboBehandelten $(p=0,01)$ [36]. Ein Abheilen der entzündeten Schleimhaut wurde bei 8 von 22 Patienten beobachtet, die in der Curcumin-Gruppe (38\%) ausgewertet wurden, verglichen mit keinem von 16 Patienten in der Placebo-Gruppe ( $p=0,043)$ [36]. Nebenwirkungen traten selten auf und waren vergleichbar zwischen den Gruppen. Curcumin kann also ein sicheres und vielversprechendes Mittel für die Behandlung von Colitis ulcerosa sein.

Inzwischen sind sehr viele verschiedene Curcuma-Präparationen und -Kapseln auf dem Markt zu finden. Es gibt jedoch keinen Hinweis darauf, dass Zusätze wie Vitamin C oder andere Vitamine oder auch das Piperazin (zumindest bei Colitis ulcerosa) irgendwelche Vorteile hätten. Das Curcuma wird dadurch unverhältnismässig teuer. Ben-Horin hatte in seiner Studie «einfaches» Gewürz-Curcuma verwendet!

\section{Fazit}

Zahlreiche Studien konnten belegen, dass Anthocyane und auch Curcuma eine antientzündliche und schützende Wirkung für den Organismus entfalten. Dies konnte sowohl in Mausversuchen als auch in einer ersten klinischen Studie bei Patienten mit Colitis ulcerosa nachgewiesen werden. Da Anthocyane und Curcuma natürliche Nahrungsbestandteile sind und somit keine Nebenwirkungen aufweisen, würden sie grundsätzlich ein ideales Therapeutikum darstellen. Allerdings bedarf es weiterer und deutlich breiter angelegter Studien, um eine abschliessende Beurteilung hinsichtlich der Wirksamkeit von Anthocyanen und Curcuma bei Patienten mit chronisch-entzündlichen Darmerkrankungen vornehmen zu können. 


\section{Literatur}

1 Langhorst J, Wulfert H, Lauche R, Klose P, Cramer H, Dobos GJ, Korzenik J: Systematic review of complementary and alternative medicine treatments in inflammatory bowel diseases. J Crohns Colitis 2015;9:86-106.

2 Crozier A, Jaganath IB, Clifford MN: Dietary phenolics: chemistry, bioavailability and effects on health. Nat Prod Rep 2009;26:10011043.

3 Cardona F, Andres-Lacueva C, Tulipani S, Tinahones FJ, Queipo-Ortuno MI: Benefits of polyphenols on gut microbiota and implications in human health. J Nutr Biochem 2013; 24:1415-1422

4 Ovaskainen ML, Torronen R, Koponen JM, Sinkko H, Hellstrom J, Reinivuo H, Mattila P: Dietary intake and major food sources of polyphenols in Finnish adults. J Nutr 2008;138: 562-566.

5 Rodriguez-Mateos A, Vauzour D, Krueger CG, Shanmuganayagam D, Reed J, Calani L, Mena P, Del Rio D, Crozier A: Bioavailability, bioactivity and impact on health of dietary flavonoids and related compounds: an update. Arch Toxicol 2014;88:1803-1853.

6 Nohynek LJ, Alakomi HL, Kahkonen MP, Heinonen M, Helander IM, Oksman-Caldentey KM, Puupponen-Pimia RH: Berry phenolics: antimicrobial properties and mechanisms of action against severe human pathogens. Nutr Cancer 2006;54:18-32.

7 Scalbert A, Williamson G: Dietary intake and bioavailability of polyphenols. J Nutr 2000; 130:2073S-2085S

8 Hooper L, Kay C, Abdelhamid A, Kroon PA, Cohn JS, Rimm EB, Cassidy A: Effects of chocolate, cocoa, and flavan-3-ols on cardiovascular health: a systematic review and meta-analysis of randomized trials. Am J Clin Nutr 2012; 95:740-751.

9 Jennings A, Welch AA, Fairweather-Tait SJ, Kay C, Minihane AM, Chowienczyk P, Jiang B, Cecelja M, Spector T, Macgregor A, Cassidy A: Higher anthocyanin intake is associated with lower arterial stiffness and central blood pressure in women. Am J Clin Nutr 2012;96: 781-788.

10 Yan X, Murphy BT, Hammond GB, Vinson JA, Neto CC: Antioxidant activities and antitumor screening of extracts from cranberry fruit (Vaccinium macrocarpon). J Agric Food Chem 2002;50:5844-5849.

11 Frankel EN, Kanner J, German JB, Parks E, Kinsella JE: Inhibition of oxidation of human low-density lipoprotein by phenolic substances in red wine. Lancet 1993;341:454-457.

12 Mauray A, Felgines C, Morand C, Mazur A, Scalbert A, Milenkovic D: Bilberry anthocyanin-rich extract alters expression of genes related to atherosclerosis development in aorta of apo E-deficient mice. Nutr Metab Cardiovasc Dis 2012;22:72-80.

13 Meloni F, Ballabio P, Gorrini M, De Amici M, Marena C, Malandrino S, Luisetti M: Effects of 3'-hydroxyfarrerol (IdB 1031), a novel flavonoid agent, on phagocyte products. Inflammation 1995;19:689-699.
14 Tsoyi K, Park HB, Kim YM, Chung JI, Shin SC, Lee WS, Seo HG, Lee JH, Chang KC, Kim $\mathrm{HJ}$ : Anthocyanins from black soybean seed coats inhibit UVB-induced inflammatory cylooxygenase- 2 gene expression and PGE2 production through regulation of the nuclear factor-kappaB and phosphatidylinositol 3-kinase/ Akt pathway. J Agric Food Chem 2008;56: 8969-8974.

15 Boivin D, Blanchette M, Barrette S, Moghrabi A, Beliveau R: Inhibition of cancer cell proliferation and suppression of TNF-induced activation of NFkappaB by edible berry juice. Anticancer Res 2007;27:937-948.

16 Rimando AM, Kalt W, Magee JB, Dewey J, Ballington JR: Resveratrol, pterostilbene, and piceatannol in vaccinium berries. J Agric Food Chem 2004;52:4713-4719.

17 Dreiseitel A, Schreier P, Oehme A, Locher S, Rogler G, Piberger H, Hajak G, Sand PG: Inhibition of proteasome activity by anthocyanins and anthocyanidins. Biochem Biophys Res Commun 2008;372:57-61.

18 Roth S, Spalinger MR, Muller I, Lang S, Rogler G, Scharl M: Bilberry-derived anthocyanins prevent IFN- $\gamma$-induced pro-inflammatory signalling and cytokine secretion in human THP-1 monocytic cells. Digestion 2014;90: 179-189.

19 Karlsen A, Paur I, Bohn SK, Sakhi AK, Borge GI, Serafini M, Erlund I, Laake P, Tonstad S, Blomhoff R: Bilberry juice modulates plasma concentration of NF- $\mathrm{kB}$ related inflammatory markers in subjects at increased risk of CVD. Eur J Nutr 2010;49:345-355.

20 Granfeldt YE, Bjorck IM: A bilberry drink with fermented oatmeal decreases postprandial insulin demand in young healthy adults. Nutr J 2011;10:57.

21 Thomasset S, Berry DP, Cai H, West K, Marczylo TH, Marsden D, Brown K, Dennison A, Garcea G, Miller A, Hemingway D, Steward WP, Gescher AJ: Pilot study of oral anthocyanins for colorectal cancer chemoprevention. Cancer Prev Res (Phila) 2009;2:625-633.

22 Osman N, Adawi D, Ahrne S, Jeppsson B, Molin G: Probiotics and blueberry attenuate the severity of dextran sulfate sodium (DSS)induced colitis. Dig Dis Sci 2008;53:2464-2473.

23 Montrose DC, Horelik NA, Madigan JP, Stoner GD, Wang LS, Bruno RS, Park HJ, Giardina C, Rosenberg DW: Anti-inflammatory effects of freeze-dried black raspberry powder in ulcerative colitis. Carcinogenesis 2011;32: 343-350.

24 Piberger H, Oehme A, Hofmann C, Dreiseitel A, Sand PG, Obermeier F, Schoelmerich J, Schreier P, Krammer G, Rogler G: Bilberries and their anthocyanins ameliorate experimental colitis. Mol Nutr Food Res 2011;55:17241729.

25 Biedermann L, Mwinyi J, Scharl M, Frei P, Zeitz J, Kullak-Ublick GA, Vavricka SR, Fried M, Weber A, Humpf HU, Peschke S, Jetter A, Krammer G, Rogler G: Bilberry ingestion improves disease activity in mild to moderate ulcerative colitis - an open pilot study. J Crohns Colitis 2013;7:271-279.
26 Roth S, Spalinger MR, Gottier C, Biedermann L, Zeitz J, Lang S, Weber A, Rogler G, Scharl M: Bilberry-derived anthocyanins modulate cytokine expression in the intestine of patients with ulcerative colitis. PLoS One 2016;11: e0154817.

27 Wang J, Ghosh SS, Ghosh S: Curcumin improves intestinal barrier function: modulation of intracellular signaling, and organization of tight junctions. Am J Physiol Cell Physiol 2017; 312:C438-C445.

28 Xu S, Jiang B, Wang H, Shen C, Chen H, Zeng L: Curcumin suppresses intestinal fibrosis by inhibition of PPAR $\gamma$-mediated epithelial-mesenchymal transition. Evid Based Complement Alternat Med 2017;2017:7876064.

29 Qiao H, Fang D, Chen J, Sun Y, Kang C, Di L, Li J, Chen Z, Chen J, Gao Y: Orally delivered polycurcumin responsive to bacterial reduction for targeted therapy of inflammatory bowel disease. Drug Deliv 2017;24:233-242.

30 Park MJ, Moon SJ, Lee SH, Yang EJ, Min JK, Cho SG, Yang CW, Park SH, Kim HY, Cho ML: Curcumin attenuates acute graft-versushost disease severity via in vivo regulations on Th1, Th17 and regulatory T cells. PLoS One 2013;8:e67171.

31 McFadden RM, Larmonier CB, Shehab KW, Midura-Kiela M, Ramalingam R, Harrison CA, Besselsen DG, Chase JH, Caporaso JG, Jobin C, Ghishan FK, Kiela PR: The role of curcumin in modulating colonic microbiota during colitis and colon cancer prevention. Inflamm Bowel Dis 2015;21:2483-2494.

32 Jobin C, Bradham CA, Russo MP, Juma B, Narula AS, Brenner DA, Sartor RB: Curcumin blocks cytokine-mediated NF-kappa B activation and proinflammatory gene expression by inhibiting inhibitory factor I-kappa B kinase activity. J Immunol 1999;163:3474-3483.

33 Venkataranganna MV, Rafiq M, Gopumadhavan S, Peer G, Babu UV, Mitra SK: NCB-02 (standardized Curcumin preparation) protects dinitrochlorobenzene-induced colitis through down-regulation of NFkappa-B and iNOS. World J Gastroenterol 2007;13:1103-1107.

34 Sugimoto K, Hanai H, Tozawa K, Aoshi T, Uchijima M, Nagata T, Koide Y: Curcumin prevents and ameliorates trinitrobenzene sulfonic acid-induced colitis in mice. Gastroenterology 2002;123:1912-1922.

35 Hanai H, Iida T, Takeuchi K, Watanabe F, Maruyama Y, Andoh A, Tsujikawa T, Fujiyama Y, Mitsuyama K, Sata M, Yamada M, Iwaoka Y, Kanke K, Hiraishi H, Hirayama K, Arai H, Yoshii S, Uchijima M, Nagata T, Koide Y: Curcumin maintenance therapy for ulcerative colitis: randomized, multicenter, double-blind, placebo-controlled trial. Clin Gastroenterol Hepatol 2006;4:1502-1506.

36 Lang A, Salomon N, Wu JC, Kopylov U, Lahat A, Har-Noy O, Ching JY, Cheong PK, Avidan B, Gamus D, Kaimakliotis I, Eliakim R, Ng SC, Ben-Horin S: Curcumin in combination with mesalamine induces remission in patients with mild-to-moderate ulcerative colitis in a randomized controlled trial. Clin Gastroenterol Hepatol 2015;13:1444-1449.e1441. 